\section{Zur Frage der Biosynthese von Gallussäure}

Von M. H. ZENK

Botanisches Institut der Universität München

(Z. Naturforschg. 19 b, 83—84 [1964] ; eingegangen am 6. November 1963)

Die Gallussäure ist in höheren Pflanzen eine der weitest verbreiteten, mengenmäßig am stärksten vertretenen und in Form ihrer Derivate technisch wichtigsten Pflanzensäure. Über ihre Biosynthese ist nur wenig bekannt. Bei Phycomyces blakesleeanus wird für die Biosynthese der Gallussäure einerseits eine Dehydrierung der 5-Dehydroshikimisäure angenommen ${ }^{1}$, andererseits läßt sich nach Befunden von BRUCKER ${ }^{2}$ die Bildung der Gallussäure bei diesem Pilz auch vom Tyrosin ausgehend formulieren; im letzteren Fall wird angenommen, daß zuerst der Phenylring oxydiert und dann die Seitenkette des Tyrosins durch $\beta$-Oxydation verkürzt wird. Mesoinosit konnte als Vorstufe für die Biosynthese von Gallussäure ausgeschlossen werden ${ }^{3}$. Die Bildungsmöglichkeit der Gallussäure aus Shikimisäure wird durch Versuche an höheren Pflanzen ${ }^{4}$ gestützt; sie zeigten, daß nach Verfütterung von Acetat, Glucose und Phenylalanin an Blätter von Geranium pyrenaicum Glucose wesentlich besser als Acetat und Phenylalanin in Gallussäure eingebaut wird.

Durch den Nachweis, daß $p$-Hydroxybenzoesäure aus Phenylalanin durch $\beta$-Oxydation der aus der Aminosäure entstandenen $p$-Cumarsäure gebildet wird ${ }^{5}$, wurden wir veranlaßt, nochmals den Einbau von Glucose, Phenylalanin und auch Tyrosin und Benzoesäure in die Gallussäure zu untersuchen. Als Objekt diente Rhus typhina, die als Gallotannin führende Gerbstoffpflanze bekannt ist ${ }^{6}$, und die sich in Vorversuchen als die zur Untersuchung günstigste Anacardiacee herausgestellt hatte.

Für die Versuche wurden ausgestanzte Blattstücke ( $1,1 \mathrm{~cm}$ Durchmesser) in Lösungen ${ }^{14} \mathrm{C}$-markierter Substanzen unter guter Belüftung 24 Stdn. im Dunkeln inkubiert. Danach wurde das Gewebe durch Einbringen in heiße $\left(95^{\circ} \mathrm{C}\right) 2-n . \mathrm{H}_{2} \mathrm{SO}_{4}$ abgetötet und der Zellinhalt 6 Stdn. bei dieser Temperatur hydrolysiert. Die Gallussäure wurde durch Ätherextraktion des Hydrolysates, Rückextraktion in gesättigter Bicarbonatlösung und nach Ansäuerung der Wasserphase durch abermalige Ätherextraktion angereichert; die weitere Reinigung erfolgte dünnschichtchromatographisch auf Kieselgel-Platten in den beiden Lösungsmitteln Benzol : Methanol : Eisessig (90:16:8; $\left.R_{f}: 0,3\right)$ und Chloroform : Äthylacetat : Ameisensäure $\left(50: 40: 10 ; R_{f}\right.$ : $0,5)$; anschließend wurde das Produkt umkristallisiert.

1 E. Haslam, R. D. Haworth u. P. F. Knowles, J. chem. Soc. [London] 1961, 1854.

2 W. Brucker, Planta 48, 627 [1957].

3 F. Weygand, W. Brucker, H. Grisebach u. E. Schulze, Z. Naturforschg. 12 b, 222 [1957].

4 E. E. Conn u. T. Swain, Chem. Ind., 1961, 592.

5 M. H. ZeNk u. G. Müller, in Vorbereitung.

6 J. v. Wiesner, Gerbstoffe, J. Cramer Verlag, Weinheim 1962.
Ein Zufügen von inaktiver Trägergallussäure erübrigte sich wegen der hohen Säurekonzentration des Gewebes (17 mg/g Frischgewicht). Die Bestimmung der Gallussäure erfolgte spektrophotometrisch und gravimetrisch; die Decarboxylierung wurde mit Kupferchromit bei $270{ }^{\circ} \mathrm{C}$ durchgeführt; Ausbeute an $\mathrm{CO}_{2}: 70$ Prozent.

Die Ergebnisse, die in Tab. 1 zusammengestellt sind, zeigen, daß unter diesen experimentellen Bedingungen und mit diesem Pflanzenmaterial Phenylalanin weitaus besser in Gallussäure eingebaut wird als Glucose-2- ${ }^{14} \mathrm{C}$ (wobei die Radioaktivität der Glucose bei direkter Einlagerung über Shikimisäure vornehmlich im $\mathrm{C}_{1}$ der Gallussäure erscheinen sollte). Benzoesäure wird nur zu einem geringen Teil hydroxyliert. Der um ca. eine Zehnerpotenz niedrigere Einbau von Tyrosin läßt sich auf die geringe Aktivität der Tyrosindesaminase (Tyrase) in dicotylen Pflanzen zurückführen ${ }^{7}$. Da nur die L-Formen der Aminosäuren von den entsprechenden Enzymen desaminiert werden, die D-Formen in höheren Pflanzen zwar metabolisch aufgenommen aber nicht racemisiert werden können ${ }^{8}$, wurde der Aufnahmewert zur Berechnung der Einbaurate halbiert.

Es ist daher wahrscheinlich, daß die Gallussäure-Biosynthese in Anacardiaceen mehr über den Phenylpropanstoffwechsel läuft, als direkt über die Dehydrierung der 5-Dehydroshikimisäure. Da der Umbau des Phenylalanins zu den aromatischen $\mathrm{C}_{7}$-Säuren über Zimtsäure ${ }^{9,5}$ mit nachfolgender Hydroxylierung des Ringes und anschließender $\beta$-Oxydation der Seitenkette erfolgt, wird folgender Biosyntheseweg der Gallussäure vorgeschlagen:

Phenylalanin $\rightarrow$ Zimtsäure $\rightarrow p$-Cumarsäure $\rightarrow$ $\rightarrow$ Kaffeesäure $\rightarrow$

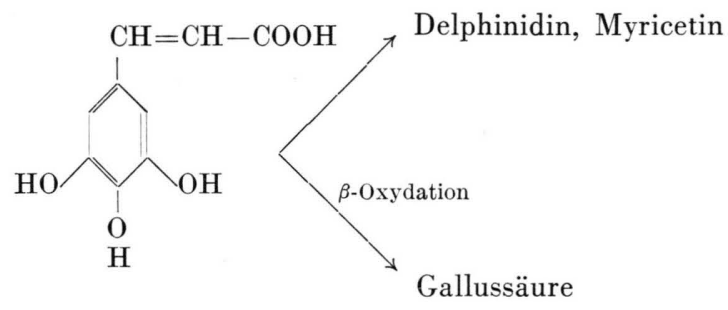

Die als Zwischenprodukt geforderte und bisher in der Natur noch nicht aufgefundene ${ }^{10}$ 3.4.5-Trihydroxyzimtsäure könnte als Thioester mit $\mathrm{CoA}$ einerseits durch $\beta$-Oxydation zu Gallussäure abgebaut werden, andererseits aber auch in das Anthocyanidin Delphinidin und das Flavonol-Aglykon Myricetin eingebaut werden. Für diese Auffassung spricht z. B. auch das gleichzeitige

7 A. C. NeIsh, Phytochem. 1, 1 [1961].

8 M. H. Zenk u. H. Scherf, Biochim. biophysica Acta [Amsterdam] 71, 737 [1963].

9 H. Grisebach u. K.-O. Vollmer, Z. Naturforschg. 18 b, 753 [1963].

10 W. Karrer, Konstitution und Vorkommen der organischen Pflanzenstoffe, Birkhäuser Verlag, Basel 1958. 
Auftreten von Myricetin und Gallotannin in der Gat tung Rhus. Die aus der $\beta$-Oxydationsspirale als Galloyl-CoA austretende aktivierte Gallussäure könnte möglicherweise die bei der Gallotannin-Biosynthese erforderliche Energie zur Knüpfung der Esterbindung zwischen Gallussäure und Glucosehydroxyl mitbringen. Diese Fragen werden weiter untersucht.

Der Deutschen Forschungsgemeins chaf danken wir für die Unterstützung dieser Arbeit.

\begin{tabular}{|c|c|c|c|c|}
\hline & \multicolumn{4}{|c|}{ Angebotene Substanzen } \\
\hline & $\begin{array}{r}\text { Glucose } \\
-2-{ }^{14} \mathrm{C}\end{array}$ & $\begin{array}{c}\mathrm{D}, \mathrm{L} \text {-Phenylalanin } \\
\quad-3-{ }^{14} \mathrm{C}\end{array}$ & $\begin{array}{l}\mathrm{D}, \mathrm{L}-\mathrm{Ty} \text { rosin } \\
\quad-3-{ }^{14} \mathrm{C}\end{array}$ & $\begin{array}{c}\text { Benzoesäure } \\
-7-{ }^{14} \mathrm{C}\end{array}$ \\
\hline $\begin{array}{l}\text { Aktivität angeboten [ipm] } \\
\text { Aktivität aufgenommen [ipm] } \\
\text { Aktivität in der Säurefraktion } \\
\text { [ipm] } \\
\text { Gesamtaktivität } \\
\text { in Gallussäure [ipm] } \\
\text { spezifische Aktivität } \\
\text { [ipm/mMol] } \\
\text { Aktivität i. d. Carboxylgruppe } \\
\text { d. Gallussäure [\%] } \\
\text { Einbaurate [\%] }\end{array}$ & $\begin{array}{l}6,3^{0} \cdot 10^{6} \\
1,12 \cdot 10^{6} \\
8,0 \cdot 10^{3} \\
2,8 \cdot 10^{2} \\
1,8 \cdot 10^{3} \\
-\frac{-}{2,5} \cdot 10^{-2}\end{array}$ & $\begin{array}{l}10,89 \cdot 10^{6} \\
5,28 \cdot 10^{6} \\
9,7 \cdot 10^{4} \\
1,3 \cdot 10^{4} \\
8,3 \cdot 10^{4} \\
92 \\
4,9 \cdot 10^{-1}\end{array}$ & $\begin{array}{l}8,94 \cdot 10^{6} \\
4,54 \cdot 10^{6} \\
1,3 \cdot 10^{4} \\
1,4 \cdot 10^{3} \\
9,0 \cdot 10^{3} \\
6,2 \cdot 10^{-2}\end{array}$ & $\begin{array}{l}5,69 \cdot 10^{6} \\
2,13 \cdot 10^{6} \\
8,84 \cdot 10^{5} \\
3,4 \cdot 10^{2} \\
2,2 \cdot 10^{3} \\
-1,6 \cdot 10^{-2}\end{array}$ \\
\hline
\end{tabular}

Tab. 1. Inkubation von $1,5 \mathrm{~g}$ Rhus typhina Blattgewebe in $25 \mathrm{ml}$ einer Lösung $10^{-4}-\mathrm{m}$. in bezug auf die markierte Vorstufe und $10^{-2}-m$. in bezug auf Citratphosphatpuffer $p_{\mathrm{H}} 5,0$. Inkubationsdauer 24 Stdn., Temperatur $21^{\circ} \mathrm{C}$. 